\title{
Femtosecond X-ray protein nanocrystallography
}

Henry N. Chapman ${ }^{1,2}$, Petra Fromme ${ }^{3}$, Anton Barty ${ }^{1}$, Thomas A. White ${ }^{1}$, Richard A. Kirian ${ }^{4}$, Andrew Aquila ${ }^{1}$, Mark S. Hunter ${ }^{3}$, Joachim Schulz ${ }^{1}$, Daniel P. DePonte ${ }^{1}$, Uwe Weierstall ${ }^{4}$, R. Bruce Doak ${ }^{4}$, Filipe R.N.C. Maia ${ }^{5}$, Andrew V. Martin ${ }^{1}$, Ilme Schlichting ${ }^{6,7}$, Lukas Lomb ${ }^{7}$, Nicola Coppola ${ }^{1}$, Robert L. Shoeman ${ }^{7}$, Sascha W. Epp ${ }^{6,8}$, Robert Hartmann ${ }^{9}$, Daniel Rolles ${ }^{6,7}$, Artem Rudenko ${ }^{6,8}$, Lutz Foucar ${ }^{6,7}$, Nils Kimmel ${ }^{10}$, Georg Weidenspointner ${ }^{11,10}$, Peter Holl ${ }^{9}$, Mengning Liang ${ }^{1}$, Miriam Barthelmess ${ }^{12}$, Carl Caleman $^{1}$, Sébastien Boutet ${ }^{13}$, Michael J. Bogan ${ }^{14}$, Jacek Krzywinski ${ }^{13}$, Christoph Bostedt $^{13}$, Saša Bajt ${ }^{12}$, Lars Gumprecht ${ }^{1}$, Benedikt Rudek ${ }^{6,8}$, Benjamin Erk ${ }^{6,8}$, Carlo Schmidt $^{6,8}$, André Hömke ${ }^{6,8}$, Christian Reich ${ }^{9}$, Daniel Pietschner ${ }^{10}$, Lothar Strüder ${ }^{6,10}$, Günter Hauser $^{10}$, Hubert Gorke ${ }^{15}$, Joachim Ullrich ${ }^{6,8}$, Sven Herrmann ${ }^{10}$, Gerhard Schaller $^{10}$, Florian Schopper ${ }^{10}$, Heike Soltau ${ }^{9}$, Kai-Uwe Kühnel ${ }^{8}$, Marc

Messerschmidt $^{13}$, John D. Bozek ${ }^{13}$, Stefan P. Hau-Riege ${ }^{16}$, Matthias Frank ${ }^{16}$, Christina Y. Hampton ${ }^{14}$, Raymond G. Sierra ${ }^{14}$, Dmitri Starodub ${ }^{14}$, Garth J. Williams ${ }^{13}$, Janos Hajdu $^{5}$, Nicusor Timneanu ${ }^{5}$, M. Marvin Seibert ${ }^{5}$, Jakob Andreasson ${ }^{5}$, Andrea Rocker ${ }^{5}$, Olof Jönsson ${ }^{5}$, Martin Svenda ${ }^{5}$, Stephan Stern ${ }^{1}$, Karol Nass ${ }^{2}$, Robert Andritschke ${ }^{10}$, Claus-Dieter Schröter ${ }^{8}$, Faton_Krasniqi ${ }^{6,7}$, Mario Bott ${ }^{7}$, Kevin E. Schmidt ${ }^{4}$, Xiaoyu Wang $^{4}$, Ingo Grotjohann ${ }^{3}$, James M. Holton ${ }^{17}$, Thomas R. M. Barends ${ }^{7}$, Richard Neutze $^{18}$, Stefano Marchesini ${ }^{17}$, Raimund Fromme ${ }^{3}$, Sebastian Schorb ${ }^{19}$, Daniela Rupp $^{19}$, Marcus Adolph ${ }^{19}$, Tais Gorkhover ${ }^{19}$, Inger Andersson ${ }^{20}$, Helmut Hirsemann ${ }^{12}$, Guillaume Potdevin $^{12}$, Heinz Graafsma ${ }^{12}$, Björn Nilsson ${ }^{12}$, and John C. H. Spence ${ }^{4}$

1. Center for Free-Electron Laser Science, DESY, Notkestrasse 85, 22607 Hamburg, Germany.

2. University of Hamburg, Luruper Chaussee 149, 22761 Hamburg, Germany. 
3. Department of Chemistry and Biochemistry, Arizona State University, Tempe, Arizona 85287-1604 USA.

4. Department of Physics, Arizona State University, Tempe, Arizona 85287 USA.

5. Laboratory of Molecular Biophysics, Department of Cell and Molecular Biology, Uppsala University, Husargatan 3 (Box 596), SE-751 24 Uppsala, Sweden.

6. Max Planck Advanced Study Group, Center for Free Electron Laser Science (CFEL), Notkestrasse 85, 22607 Hamburg, Germany.

7. Max-Planck-Institut für medizinische Forschung, Jahnstr. 29, 69120 Heidelberg, Germany.

8. Max-Planck-Institut für Kernphysik, Saupfercheckweg 1, 69117 Heidelberg, Germany.

9. PNSensor GmbH, Otto-Hahn-Ring 6, 81739 München, Germany.

10. Max-Planck-Institut Halbleiterlabor, Otto-Hahn-Ring 6, 81739 München, Germany.

11. Max-Planck-Institut für extraterrestrische Physik, Giessenbachstrasse, 85741

Garching, Germany.

12. Photon Science, DESY, Notkestrasse 85, 22607 Hamburg, Germany.

13. LCLS, SLAC National Accelerator Laboratory, 2575 Sand Hill Road. Menlo Park, CA 94025, USA.

14. Stanford PULSE Institute, SLAC National Accelerator Laboratory, 2575 Sand Hill Road. Menlo Park, CA 94025, USA.

15. Forschungszentrum Jülich, Institut ZEL, 52425 Jülich, Germany. 
16. Lawrence Livermore National Laboratory, 7000 East Avenue, Mail Stop L-211, Livermore, CA 94551, USA.

17. Advanced Light Source, Lawrence Berkeley National Laboratory, Berkeley, California 94720, USA.

18. Department of Chemistry, Biochemistry and Biophysics, University of Gothenburg, SE-405 30 Gothenburg, Sweden

19. Institut für Optik und Atomare Physik, Technische Universität Berlin, Hardenbergstrasse 36, 10623 Berlin, Germany.

20. Department of Molecular Biology, Swedish University of Agricultural Sciences, Uppsala Biomedical Centre, Box 590, S-751 24 Uppsala, Sweden.

X-ray crystallography provides the vast majority of macromolecular structures, but the success of the method relies upon growing crystals of sufficient size. In conventional measurements, the necessary increase in $\mathrm{X}$-ray dose to record data from crystals that are too small leads to extensive damage before a diffraction signal can be recorded ${ }^{1-3}$. It is particularly challenging to obtain large welldiffracting crystals of membrane proteins, for which less than 300 unique structures have been determined despite their importance in all living cells. Here we present a method for structure determination where single-crystal X-ray diffraction "snapshots" are collected from a fully hydrated stream of nanocrystals using femtosecond pulses from a hard X-ray free-electron laser, the Linac Coherent Light Source (LCLS) ${ }^{4}$. We prove this concept with nanocrystals of Photosystem I, one of the largest membrane protein complexes ${ }^{5}$. Over 3 million diffraction patterns were collected in this study, and a three-dimensional (3D) data set was assembled from individual Photosystem I nanocrystals $(\sim 200 \mathrm{~nm}$ to $2 \mu \mathrm{m}$ in size). We mitigate the problem of radiation damage in crystallography by using 


\section{pulses briefer than the time-scale of most damage processes ${ }^{6}$. This offers a new approach to structure determination of macromolecules that do not yield crystals of sufficient size for studies using conventional radiation sources or are particularly sensitive to radiation damage.}

From the beginning, radiation damage has limited resolution in biological imaging utilising electrons or X-rays ${ }^{2}$. With the recent invention of the femtosecond X-ray laser, an opportunity has arisen to break the nexus between radiation dose and spatial resolution. It has been proposed that femtosecond X-ray pulses can be used to out-run even the fastest damage processes by using single pulses so brief that they terminate before the manifestation of damage to the sample ${ }^{6}$. Experiments at the FLASH freeelectron laser (FEL) confirmed the feasibility of "diffraction before destruction" at resolution lengths down to $60 \AA$ on test samples fixed on silicon nitride membranes ${ }^{7}$. It was predicted that the irradiance (or power density) of focused pulses from a hard X-ray FEL such as LCLS would be sufficient to produce diffraction patterns at near-atomic resolution ${ }^{6}$.

We demonstrate here that this notion of "diffraction-before-destruction" operates at sub-nanometre resolution, using the membrane protein Photosystem I as a model system, and establish an approach to structure determination based on X-ray diffraction data from a stream of nanocrystals ${ }^{6,8}$. Membrane proteins play a central role in the function of cells and viruses, yet our knowledge of the structure and dynamics responsible for their function remains limited. Photosystem I is a large membrane protein complex ( $1 \mathrm{MDa}$ molecular mass, 36 proteins, 381 cofactors) that acts as a biosolar energy converter in the process of oxygenic photosynthesis. Its crystals display the symmetry of space group P6 $6_{3}$, with unit cell parameters $a=b=281 \AA, c=165 \AA$ and consist of $78 \%$ solvent by volume. We show that diffraction data can be recorded from these fragile protein nanocrystals before destruction occurs. Furthermore, we 
demonstrate that structure factors can be extracted from the "partial" reflections of tens of thousands of single crystal diffraction "snapshots," showing that interpretable highquality 3D structure factor data can be obtained from a suspension of sub-micrometre crystals.

Our experimental setup (see Fig. 1 and Methods), records single-crystal diffraction data from a stream of crystals carried in a $4-\mu \mathrm{m}$ diameter continuous liquid water jet ${ }^{9}$ that flows across the focused LCLS X-ray beam in vacuum at $10 \mu \mathrm{l} /$ minute. In contrast to cryo-electron microscopy ${ }^{10,11}$ or standard crystallography on microcrystals ${ }^{3}$, which require cryo-cooling, these data were collected on fully hydrated 3D nanocrystals. The crystal located in the interaction region when an X-ray pulse arrives gives rise to a diffraction pattern that is detected on a set of two low-noise X-ray pnCCD modules $^{12}$, and read out before the arrival of the next pulse at the FEL repetition rate of $30 \mathrm{~Hz}$, or 1,800 patterns per minute. The photon energy of the X-ray pulses was $1.8 \mathrm{keV}$ (6.9 A wavelength), with more than $10^{12}$ photons per pulse at the sample and pulse durations of 10, 70, and $200 \mathrm{fs}$ (ref 13). An X-ray fluence of $900 \mathrm{~J} / \mathrm{cm}^{2}$ was achieved by focusing the FEL beam to $7 \mu \mathrm{m}$ full-width at half maximum, corresponding to a sample dose of up to $700 \mathrm{MGy}$ per pulse (calculated by the program RADDOSE ${ }^{14}$ ) and a peak power density in excess of $10^{16} \mathrm{~W} / \mathrm{cm}^{2}$ at $70 \mathrm{fs}$ duration. In contrast, the typical tolerable dose in conventional X-ray experiments is only about $30 \mathrm{MGy}$ (ref. 1). A single LCLS X-ray pulse destroys any solid material placed in this focus, but the stream replenishes the vaporised sample before the next pulse.

The front detector module located close to the interaction region recorded highangle diffraction to a resolution of $8.5 \AA$, whereas the rear module intersected diffraction covering $4000 \AA$ to $100 \AA \AA$ resolution. We observed diffraction from crystals smaller than 10 unit cells on a side, as determined by examining the data recorded on the rear pnCCDs shown in Fig. 2. A crystal with a side length of $N$ unit cells gives rise 
to diffraction features that are $1 / N$ finer than the Bragg spacing (or $N-2$ fringes between neighbouring Bragg peaks), providing a simple way to determine the projected size of the nanocrystal. Images of crystal shapes are shown in Fig. 2, obtained using an iterative phase retrieval method ${ }^{15,16}$. The 3D Fourier transform of the crystal shape is repeated on every reciprocal lattice point. However, the diffraction condition of lattice points is usually not exactly satisfied so each recorded Bragg spot represents a particular "slice" of the Ewald sphere through the shape transform, giving a variety of Bragg spot profiles in a pattern, apparent in Fig. 2. The summed counts in each Bragg spot underestimates the underlying structure factor square modulus, representing a "partial reflection."

Figure 3 (a) shows strong single crystal diffraction to the highest angles of the front detector. The nanocrystal shape transform is also apparent in many patterns at the high angles detected by the front detector, giving significant measured intensities between Bragg peaks as is noticeable in Fig. S3 (a). These mid-Bragg intensities oversample the molecular transform, providing a potential route to phasing of the pattern $^{17,18}$.

In conventional crystallography, the "full" Bragg reflection is determined to high precision, for example by integrating counts as the crystal is rotated such that these reflections pass through the diffraction condition. By indexing individual patterns and then summing counts in all "partial reflections" for each index, we performed a Monte Carlo integration over the reciprocal space volume of the Bragg reflection and the distribution of crystal shapes, orientations, and variations in X-ray pulse fluence. This procedure converges to the square of the structure factor moduli ${ }^{18}$. We found that over $13 \%$ of diffraction patterns with 10 or more spots could be consistently indexed using the programs MOSFLM ${ }^{19}$ and $\operatorname{DirAx}^{20}$ (see Full Methods). Merged intensities at $70 \mathrm{fs}$ pulse duration are presented as a precession-style image of the [001] zone axis in Fig. 
3(b) (see also Figs. S3 and S4 in Supplemental Information). The reliability of this approach was tested by comparing the LCLS merged data with data collected at $100 \mathrm{~K}$ with $12.4 \mathrm{keV}$ synchrotron radiation from a single crystal of Photosystem I cryopreserved in $2 \mathrm{M}$ sucrose. These datasets show good agreement, with a difference metric $R_{\text {iso }}=22.1 \%$ computed over the entire resolution range and below $13 \%$ in the middle resolution shells; see Table S1 for detailed statistics.

To complete our proof of principle, we conducted a rigid-body refinement of the published Photosystem I structure (1JB0) against the nanocrystal structure factors, yielding $R / R_{\text {free }}=0.25 / 0.23$. A representative region of the $8.5 \AA 2 m F_{\text {obs }}-D F_{\text {calc }}$ electron density map (see Full Methods) from the LCLS data set is shown in Fig. 3 (c). The electron density map shows details as expected at this resolution, including transmembrane helices, membrane extrinsic features and some loop structures. For comparison, the electron density refined from the $12.4 \mathrm{keV}$ single crystal dataset truncated to $8.5 \AA$ resolution is given in Fig. 3 (d).

The dose of $700 \mathrm{MGy}$ corresponds to a $K$-shell photoabsorption of 3\% of all carbon atoms in the protein. This energy is subsequently released by photoionisation and Auger decay, followed by a cascade of lower-energy electrons caused by secondary ionisations, taking place on a 10 to $100 \mathrm{fs}$ timescale ${ }^{21}$. Using a model of the plasma dynamics $^{22,23}$ we calculated that, by the end of a 100 fs pulse, each atom of the crystal was ionised once, on average, and that motion of nuclei had begun. This is expected to give rise to a decrease in Bragg amplitudes, similar to an increase in a Debye-Waller temperature factor ${ }^{24}$. We studied the effects of the initial ionisation damage on the diffraction of Photosystem I nanocrystals by collecting a series of data sets at pulse durations of $10 \mathrm{fs}, 70 \mathrm{fs}$, and $200 \mathrm{fs}$. The $10 \mathrm{fs}$ pulses were produced with lower pulse energy: $\sim 10 \%$ of the total number of photons of the longer pulses ${ }^{13}$, or 70 MGy dose. Plots of the scattering strength of the crystals versus resolution are depicted in Fig. 4, by 
selecting and summing Bragg spots from more than 66,000 patterns for each of the three pulse durations measured. The $10 \mathrm{fs}$ and $70 \mathrm{fs}$ traces are very similar, indicating that these pulses are short enough to overcome radiation damage at the observed $8.5 \AA$ resolution. For $200 \mathrm{fs}$ pulses there is a decrease in scattering strength at resolutions beyond $25 \AA$, indicating disordering on this longer timescale. The highest resolution Bragg peaks for the 200 fs pulses were not broadened or shifted relative to the shortduration datasets, which indicates there was no strain or expansion of the lattice, respectively.

Our next step is to improve resolution by using shorter wavelengths X-rays. Resolution may ultimately be limited by X-ray pulse fluence, the ultrafast radiation damage, and intrinsic disorder within the nanocrystals themselves. Recent experiments ${ }^{21}$ at LCLS indicate a brief saturation of the X-ray photoabsorption of atoms in a tightlyfocused pulse, resulting in a decrease in photoionisation damage on a $20 \mathrm{fs}$ timescale, without reducing the scattering cross sections that give rise to the diffraction pattern ${ }^{22}$. Planned beamlines at LCLS aim to achieve up to a $10^{5}$ fold increase in pulse irradiance by tighter focusing, allowing data collection with low-fluence $10 \mathrm{fs}$ pulses or pulses of even shorter duration ${ }^{25}$. This provides a route to further reduce radiation damage and may allow measurements on even smaller nanocrystals or objects approaching a single molecule ${ }^{6}$. As this limit is approached, the ordering of the nanocrystals will become increasingly irrelevant, as each crystal may be treated as a single object and the "disorder" that conventionally leads to reduced resolution will simply manifest itself as shot-to-shot variability, providing information about not just the average structure, but the range of dynamically accessible conformations.

Data are collected on fully hydrated nanocrystals without cryogenic cooling. We expect that the results presented here will open up new avenues for crystallography, using X-ray laser pulses that are so short that only negligible X-ray induced radiation 
damage occurs during data collection. Significant improvements in sample utilisation are expected by exploiting higher X-ray repetition rate, or by slowing the liquid flow. For example, the generation of liquid droplets at a rate that matches the LCLS X-ray pulses, using inkjet technologies, would dramatically decrease the total required sample volume by a factor of 25,000 , requiring less than $0.4 \mu \mathrm{l}$ of nanocrystal suspension in our particular case of Photosystem I. Further efficiency gains would result from indexing and merging a greater proportion of patterns into the 3D dataset, which may be achieved by applying methods for merging continuous diffraction patterns of single molecules ${ }^{26,27}$ or by employing "postrefinement" ${ }^{\text {"28 }}$ to obtain accurate structure factor estimates from fewer diffraction patterns. These methods will also remove the twinning ambiguity that exists in our current indexing scheme. Our method also has potential application to the study of chemical reactions, such as the processes in photosynthesis or enzymatic reactions.

\section{Methods Summary}

Measurements were performed using the "CFEL ASG multi purpose" (CAMP) instrument $^{12}$ on the Atomic, Molecular and Optical Science (AMO) beamline ${ }^{29}$ at the LCLS $^{4}$. Diffraction data were recorded at the LCLS repetition rate of $30 \mathrm{~Hz}$ with a set of two movable high-frame-rate low-noise X-ray pnCCD detector units ${ }^{12}$. The front detector, located $68 \mathrm{~mm}$ from the jet, accepts scattering angles up to $47.9^{\circ}$ corresponding to a resolution of $8.5 \AA$ at $6.9 \AA$ wavelength. The rear unit was located $564 \mathrm{~mm}$ from the jet to record finer sampling of the diffraction pattern at low angles.

The liquid jet is emitted from a $40-\mu \mathrm{m}$ inner-diameter capillary and focused by a coaxial flow of gas to about $4-\mu \mathrm{m}$ diameter $^{9}$, flowing at $10 \mu \mathrm{l} /$ minute. The narrow jet diameter confines the crystals to pass through the most intense part of the focused X-ray beam. Clogging of nanocrystals in the capillary is avoided, and the coaxial gas sheath prevents freezing of the liquid in the vacuum environment. A micropore filter in the 
fluid delivery line was used to restrict the size of the Photosystem I nanocrystals to smaller than $2 \mu \mathrm{m}$. The suspension was diluted to observe a crystal "hit rate" of $20 \%$ (see Fig. S2) in order to reduce the occurrence of double hits. The concentration of observed crystals was therefore 0.2 per illuminated volume of $4 \times 4 \times 13 \mu \mathrm{m}^{3}$, or about $10^{9}$ crystals $/ \mathrm{ml}$. The overall protein concentration after dilution of the suspension was $1 \mathrm{mg} / \mathrm{ml}(1 \mu \mathrm{M}$ of the Photosystem I trimer), and a complete set of structure factors was obtained from 1.85 million X-ray pulses.

Diffraction peaks from the 70 fs data were identified and indexed, and combined into a set of 3D structure factors comprising of 3,379 unique reflections from 2,424,394 spots. Statistics of the merged data are given in Table S1.

\section{References}

1. Owen, R. L., Rudino-Pinera, E. \& Garman, E. F. Experimental determination of the radiation dose limit for cryocooled protein crystals. Proc. Natl. Acad. Sci. U.S.A. 103, 4912-4917 (2006).

2. Henderson, R. The potential and limitations of neutrons, electrons and X-rays for atomic resolution microscopy of unstained biological molecules. Q.Rev. Biophys. 28, 171-193 (1995).

3. Riekel, C. Recent developments in microdiffraction on protein crystals. J. Synchr. Rad. 11, 4-6 (2004).

4. Emma, P. et al., First lasing and operation of an Ångström-wavelength free-electron laser. Nature Phot. 4, 641-647 (2010).

5. Jordan P. et al. Three-dimensional structure of cyanobacterial photosystem I at 2.5 angstrom resolution. Nature 411, 909-917 (2001). 
6. Neutze, R., Wout R., van der Spoel, D., Weckert, E. \& Hajdu, J. Potential for biomolecular imaging with femtosecond X-ray pulses. Nature 406, $752-757$ (2000).

7. Chapman, H.N. et al. Femtosecond time-delay X-ray holography. Nature 448, 676679 (2007).

8. Spence, J. C. H. \& Doak, R. B. Single molecule diffraction. Phys. Rev. Lett. 92, 198102 (2004).

9. DePonte D. P. et al. Gas dynamic virtual nozzle for generation of microscopic droplet streams. J. Phys. D 41, 195505 (2008).

10. Henderson, R. et al. Model for the structure of bacteriorhodopsin based on highresolution electron cryo-microscopy. J. Mol. Biol. 213, 899-929 (1990).

11. Wang, D. N. \& Kühlbrandt, W. High-resolution electron crystallography of lightharvesting chlorophyll a/b-protein complex in three different media. J. Mol. Biol. 217, 691-699 (1991).

12. Strüder, L. et al. Large-format, high-speed, x-ray pnCCDs combined with electron and ion imaging spectrometers in a multipurpose chamber for experiments at 4 th generation light sources. Nucl. Instrum. Meth. Phys. Res. A 614, 483 - 496 (2010). 13. Ding Y. et al. Measurements and simulations of ultralow emittance and ultrashort electron beams in the linac coherent light source. Phys. Rev. Lett., 102, 254801 (2009). 14. Paithankar, K. S., Owen, R. L. \& Garman, E. F. Absorbed dose calculations for macromolecular crystals: improvements to RADDOSE. J. Syn. Rad. 16, 152-162 (2009).

15. Marchesini S. et al. X-ray image reconstruction from a diffraction pattern alone. Phys. Rev. B 68, 140101 (2003).

16. Robinson, I. K. \& Harder R. Coherent X-ray diffraction imaging of strain at the nanoscale. Nature Mat. 8, 291 (2009). 
17. Sayre, D. Some implications of a theorem due to Shannon. Acta Cryst 5, 843 (1952).

18. Kirian, R. et al. Femtosecond protein nanocrystallography - data analysis methods. Opt. Exp. 18, 5713 (2010).

19. Leslie, A. G. The integration of macromolecular diffraction data. Acta Cryst D 62 , $48-57$ (2006).

20. Duisenberg, A. J. M. Indexing in single-crystal diffractometry with an obstinate list of reflections. J. Appl. Cryst. 25, 92-96 (1992).

21. Young L. et al. Femtosecond electronic response of atoms to ultra-intense X-rays, Nature 466, 56-61 (2010).

22. S. P. Hau-Riege, London, R. A. \& Szoke, A. Dynamics of biological molecules irradiated by short x-ray pulses. Phys. Rev. E 69, 051906 (2004).

23. Bergh, M., Huldt, G., Timneanu, N., Maia, F. R. N. C. \& Hajdu, J. Feasibility of imaging living cells at subnanometer resolutions by ultrafast x-ray diffraction. Q. Rev. Biophys. 41, 181- 204 (2008).

24. Willis, B. \& Pryor, A. Thermal vibrations in crystallography. Cambridge University Press (1975).

25. Emma, P. et al. Femtosecond and subfemtosecond x-ray pulses from a selfamplified spontaneous-emission based free-electron laser. Phys. Rev. Lett. 92, 074801 (2004).

26. Loh, N.-T. D. \& Elser, V. Reconstruction algorithm for single-particle diffraction imaging experiments. Phys. Rev. E 80026705 (2009).

27. Fung, R., Shneerson, V., Saldin, D. K. \& Ourmazd, A. Structure from fleeting illumination of faint spinning objects in flight. Nat. Phys. 5, 64-67 (2008).

28. Rossmann, M.G., Leslie A.G., Sherin S.A. \& Tsukihara T. Processing and Postrefinement of oscillation camera data. J. Appl Cryst. 12, 570 - 581 (1979). 
29. Bozek, J. D. AMO instrumentation for the LCLS X-ray FEL, Euro. Phys. J. Special Topics 169, 129-132 (2009).

30. DePonte, D. P. et al. SEM imaging of liquid jets. Micron 40507 - 509 (2009).

Supplementary Information is linked to the online version of the paper at www.nature.com/nature.

\section{Acknowledgements}

Experiments were carried out at the Linac Coherent Light Source and the Advanced Light Source, both national user facilities operated by Stanford University and the University of California (respectively) on behalf of the U.S. Department of Energy (DOE), Office of Basic Energy Sciences. We acknowledge support from DOE through the PULSE Institute at the SLAC National Accelerator Laboratory and by Lawrence Livermore National Laboratory under Contract DE-AC52-07NA27344, the Center for BioInspired Solar Fuel Production, an Energy Frontier Research Center funded by the U.S. Department of Energy, Office of Basic Energy Sciences (award DE-SC0001016), the Hamburg Ministry of Science and Research and Joachim Herz Stiftung as part of the Hamburg Initiative for Excellence in Research (LEXI) and the Hamburg School for Structure and Dynamics, the Max Planck Society for funding the development and operation of the CAMP instrument within the ASG at CFEL, National Science Foundation (awards 0417142 and MCB-1021557), National Institute of Health (awards 1R01GM09558301 and 1U54GM094625-01), the Swedish Research Council, the Swedish Foundation for International Cooperation in Research and Higher Education, Stiftelsen Olle Engkvist Byggmästare, the DFG Cluster of Excellence at the Munich Centre for Advanced Photonics, CBST at UC under Cooperative Agreement No. PHY 0120999. We acknowledge discussions with Michael Rossmann, Edward Snell, Robert Stroud, Axel Brunger, and thank the technical help of Britt Hedman, Eric Gullikson, Frank Filsinger, Andrej Berg, Helmut Mahn, and Christian Kaiser, and the staff of the LCLS for their support in carrying out these experiments. 
Author contributions H.N.C. and J.C.H.S conceived the experiment, which was designed with P.F., A.B., R.A.K., J.S., D.P.D., U.W., R.B.D., S.Bo., M.J.B., D.S., I.S., S.M. and J.H. The CAMP instrument was the responsibility of S.W.E., R.H., D.R., A.R., C.Sch, L.F., N.K., P.H., B.R., B.E., A.H., Ch.R., D.P., G.W., L.S., G.H., H.G., J.U., I.S., S.H., G.Sch., F.Sch., H.S., K.-U.K., R.A., K.-D.S., F.K., Ma.B., S.Sch., D.Ru., M.A., T.G., H.H., L.G., G.P., H.G., and B.N. who designed and set up the instrument and/or developed and operated the pnCCD detectors. C.B., J.B., and M.M. set up and aligned the beamline. P.F., M.S.H., and I.G. prepared samples, R.B.D., D.P.D., U.W., J.C.H.S., P.F., L.L., and R.L.S. developed and operated the sample delivery system, H.N.C., A.B., A.A., J.S., D.P.P., U.W., R.B.D., S.Ba., M.J.B., L.G., J.H., M.M.S., N.T., J.A., S.St., and J.C.H.S. developed diffraction instrumentation, M.B., M.L., A.B., and K.N. designed and/or fabricated calibration samples. J.K., S.H.-R., A.B., H.N.C., J.S. and A.V.M. characterised the focus. H.N.C., J.C.H.S., P.F., A.B., T.A.W., R.A.K., A.A., J.S., D.P.D., U.W., R.B.D., I.S., N.C., R.L.S., M.S.H., L.L., M.B., S.W.E., R.H., D.R., A.R., M.L., C.B., J.U., L.F., J.D.B., M.M., M.F., C.Y.H., R.G.S., G.J.W., A.R., M.S. O.J., I.A. and J.H. carried out the experiment. A.B., T.A.W., R.A.K., A.A., F.R.N.C.M., A.V.M., L.L., T.R.M.B., N.C., L.F., N.K., R.N., G.W., P.H., C.C., J.M.H., I.S., J.H., H.N.C. and J.C.H.S. analysed the data. A.V.M. performed the Bragg shape phase retrieval. T.A.W. and R.A.K. merged the 3D data. R.F. collected and evaluated the reference data set, R.A.K., T.A.W. J.M.H. and R.F. refined the structure and calculated the electron density maps, H.N.C., P.F., J.C.H.S., and I.S. wrote the manuscript with discussion and improvements from all authors.

Author information The authors declare no competing financial interests. Correspondence and requests for materials should be addressed to H.N.C. (e-mail: henry.chapman@desy.de).

Additional information The authors declare no competing financial interests. 
Figure 1. Schematic diagram of femtosecond nanocrystallography. Nanocrystals flow in their buffer solution in a gas-focused $4-\mu \mathrm{m}$ diameter jet, at a velocity of $10 \mathrm{~m} \mathrm{~s}^{-1}$, perpendicular to the pulsed X-ray free-electron laser beam that is focused on the jet. The insert shows an environmental scanning electron micrograph of the nozzle, flowing jet, and focusing gas ${ }^{30}$. Two pairs of high-frame rate pnCCD detectors ${ }^{12}$ record low and high-angle diffraction from single X-ray FEL pulses, at the FEL repetition rate of $30 \mathrm{~Hz}$. Crystals arrive at random times and orientations in the beam, and the probability of hitting one is proportional to the crystal concentration.

Figure 2. Coherent crystal diffraction. (a-d) Low-angle diffraction patterns recorded on the back pnCCDs, revealing coherent diffraction from the shape of the Photosystem I nanocrystals, displayed on a logarithmic false colour scale. The Miller indices of the peaks in (a) were identified from the corresponding high-angle pattern. In (c) we can count 7 fringes in the $b^{*}$ direction, corresponding to 9 unit cells or $250 \mathrm{~nm}$. The insert in each panel shows the real-space image of the nanocrystal, determined by phase retrieval (using the Shrinkwrap algorithm ${ }^{15}$ ) of the circled coherent Bragg shape transform.

Figure 3. Diffraction intensities and electron density of Photosystem I. (a) Diffraction pattern recorded on the front pnCCDs with a single 70 fs pulses after background subtraction and correction of saturated pixels. Some peaks are labelled with their Miller indices. The resolution in the lower detector corner is 8.5 A. (b) Precession-style pattern of the [0 0 1] zone for Photosystem I obtained from merging femtosecond nanocrystal data from over 15,000 nanocrystal patterns. A region of the $2 m F_{\text {obs }}-D F_{\text {calc }}$ electron density map at 1.0 $\sigma$ calculated from the 70 fs data (c), and from conventional synchrotron data 
truncated to $8.5 \AA$ resolution, collected at a temperature of $100 \mathrm{~K}$ (d) (see Full Methods).

Figure 4. Pulse duration dependence of diffraction intensities. Plot of the integrated Bragg intensities of Photosystem I nanocrystal diffraction as a function of photon momentum transfer $q=4 \pi / \lambda \sin \theta=2 \pi / d$, for pulse durations of $10 \mathrm{fs}, 70 \mathrm{fs}$, and $200 \mathrm{fs}$. Averages were obtained by isolating Bragg spots from $97,883,805,311$, and 66,063 patterns, respectively, normalised to pulse fluence. Errors are indicated by the thickness of the lines. The decrease in irradiance for 200 fs pulses and $d<25 \AA$ indicates radiation damage at these long pulses, which is not apparent for 70 fs pulses and shorter. 


\section{Full Methods}

\section{Experimental setup}

The experiments were performed at $\mathrm{LCLS}^{4}$ at SLAC at the AMO (Atomic, Molecular and Optical Science) beamline ${ }^{29}$ in vacuo in the CFEL-ASG Multi-Purpose (CAMP) endstation $^{12}$. X-ray pulses, generated at a repetition rate of $30 \mathrm{~Hz}$, were focused to spot of $7-\mu \mathrm{m}$ full width at half maximum (13- $\mu \mathrm{m}$ full width at $10 \%$ maximum irradiance) with a pulse fluence of $900 \mathrm{~J} / \mathrm{cm}^{2}$, corresponding to a peak power density (irradiance) in excess of $10^{16} \mathrm{~W} / \mathrm{cm}^{2}$ at 70 -fs duration. The pnCCD detectors were read out, digitised, and stored at the $30 \mathrm{~Hz}$ rate of the delivered LCLS pulses. Each detector panel consists of $512 \times 1024$ pixels of $75 \times 75 \mu \mathrm{m}^{2}$. The back detectors, located $564 \mathrm{~mm}$ from the jet, record low-angle scattering from $0.1^{\circ}$ to $4.0^{\circ}$ in the vertical scattering plane, and the front detectors, located $68 \mathrm{~mm}$ from the jet, cover $4.6^{\circ}$ to $40.5^{\circ}$ in the same vertical plane. The largest scattering angle magnitude accepted by the front detector was $47.9^{\circ}$, corresponding to a resolution $d$ of $8.5 \AA$ at $6.9 \AA$ wavelength. X-ray fluorescence from the water jet was filtered by an $8-\mu \mathrm{m}$ thick polyimide film in front of the pnCCDs.

A liquid microjet ${ }^{8,9}$ was used to inject the nanocrystal suspension into the FEL beam at a flow rate of $10 \mu 1 /$ minute. The microjet was emitted from a $40-\mu \mathrm{m}$ diameter capillary, and focused down to a 4- $\mu \mathrm{m}$ diameter column by a coaxial flow of helium. The X-ray attenuation in the water was at most $30 \%$. The interaction region of the $\mathrm{X}$ rays and crystals is located in the continuous liquid column, prior to the Rayleigh breakup of the jet into drops, so that most of the X-ray scattering from the liquid is confined to a narrow vertical streak in reciprocal space.

Crystallisation conditions of Photosystem I nanocrystals were established by determining the phase diagrams ${ }^{31,32}$. Nanocrystals were grown in batch at $10 \mathrm{mg} / \mathrm{ml}$ protein concentration $(30 \mu \mathrm{M}$ P700 $=10 \mu \mathrm{M}$ Photosystem I trimer $)$ and low ionic 
strength ( $8 \mathrm{mM} \mathrm{MgSO}_{4}, 5 \mathrm{mM}$ MES pH 6.4 and $0.02 \%$ beta-dodecylmaltoside) at $4{ }^{\circ} \mathrm{C}$. The Photosystem I nanocrystals were then suspended in harvesting buffer (5mM MES pH 6.4 and $0.02 \% \beta$-dodecylmaltoside) to establish a protein concentration of $1 \mathrm{mg} / \mathrm{ml}$. The crystal suspension was filtered through $2 \mu \mathrm{m}$ cut-off filters (Inline filter, Upchurch) and stored at $4^{\circ} \mathrm{C}$ until use in the experiment.

The nanocrystals are needles of hexagonal cross section, with the long axis of the needle along the $c$ axis and an aspect ratio (length to maximum diameter of hexagon) ranging from 1:1 to $2: 1$, as determined from reconstructing single-shot views of the whole crystal from their shape transforms. For example, Fig. 2 (a) shows a view of the crystal almost perpendicular to the $c$ axis where we reconstruct a shape of aspect ratio 1.6:1. A view down the $c$ axis (Fig. 2 c) shows the hexagonal profile. Large millimetre sized crystals of Photosystem I have an aspect ratio up to 5:1, which is seen to decrease with decreasing crystal size.

The nanocrystal suspension was introduced directly into the microjet via a sample loop (see Fig. S1). A micropore filter in the fluid delivery line was used to restrict the size of the nanocrystals to smaller than $2 \mu \mathrm{m}$. The suspension was diluted by an amount to observe a crystal "hit rate" of $20 \%$ (see Fig. S2), in order to minimise the occurrence of double hits. The observed concentration of crystals was therefore 0.2 per illuminated volume of $4 \times 4 \times 13 \mu \mathrm{m}^{3}$, or $10^{9}$ crystals $/ \mathrm{ml}$. The overall Photosystem I protein concentration after dilution was $1 \mathrm{mg} / \mathrm{ml}$, and a complete set of structure factors was obtained from 1.85 million X-ray pulses, or $10 \mathrm{mg}$ of protein. With the current setup, At the $30 \mathrm{~Hz}$ X-ray pulse rate less than $0.004 \%$ of the continuously flowing solution is exposed to the X-ray beam, so only one in 25,000 nanocrystals was actually hit by an X-ray pulse.

Details of the acquisition of diffraction patterns and the primary data reduction are given in the Supplemental Methods section. 


\section{D Merging of Intensities}

Peaks in the processed patterns were located in each pattern using the algorithm of Zaefferer $^{33}$, and their locations were mapped into three dimensions according to the curvature of the Ewald sphere, the calibrated detector geometry and the X-ray wavelength. The 3D peak locations for each pattern in turn were presented to the autoindexing program DirAx ${ }^{20}$. If DirAx succeeded in finding a unit cell for the peaks, linear combinations of the cell basis vectors were checked for correspondence with the Photosystem I unit cell from the Protein Databank (1JB0) $)^{5}$ If a match was found, pixel intensities were summed within a circle of ten-pixel radius centred on the pixel closest to each located Bragg condition. Patterns were rejected if less than $10 \%$ of the detected peaks were accounted for by unit cell parameters from DirAx. Out of 1.85 million recorded patterns we identified 112,725 as hits (greater than 10 detected peaks) and 15,445 were successfully indexed. New peak finding and indexing algorithms are under development and are expected to significantly increase the number of patterns which can be indexed, thereby also further reducing the number of protein crystals required for a useful data set. The variation of pixel solid angle across the detector plane was accounted for, as was polarisation of the X-ray beam assuming complete horizontal polarisation. A list of reflection indices and intensities was produced for each individual diffraction pattern and merging was performed by taking the mean value for the intensity of each unique reflection. Since the indexing algorithm makes use of the positions of the peaks but not their intensities, it was unable to distinguish between crystal orientations related by the symmetry of the lattice. Since the symmetry of the lattice is higher than that of the actual structure of Photosystem I, an ambiguity exists where each pattern could correspond to one of two possible orientations. For programmatic convenience, these data (with actual space group symmetry $\mathrm{P} 6_{3}$ ) were merged as $\mathrm{P}_{3} 22$ and treated as though merohedrally twinned during refinement (see below). A 3D rendering of the final full dataset is shown in Fig. S4. 


\section{Data Quality}

Metrics of the merged data quality are shown in Table S1 and discussed in the Supplemental Information. We carried out a rigid-body refinement of the published Photosystem I structure (1JB0) to the merged structure factors using the program REFMAC $^{34}$ in twin mode. The refinement $R / R_{\text {free }}$ values were $0.25 / 0.23$. The $2 m F_{\text {obs- }}$ $D F_{\text {calc }}$ electron density map ${ }^{35}$ at $8.5 \AA$ resolution is depicted in Fig. 3 (c). The corresponding $2 m F_{\text {obs }}-D F_{\text {calc }}$ electron density map from the conventional synchrotron data, truncated to $8.5 \AA$ A resolution is depicted in Fig. 3 (d). The electron density maps show the large subunits PsaA and PsaB, as well as the membrane extrinsic subunits. The transmembrane helices, and even some loop structures, are clearly visible. In these figures the ribbon representation of the protein model is shown in yellow and the atoms of three iron-sulfur clusters are depicted in red.

\section{Methods References:}

31. Fromme, P. \& Grotjohann I. in Membrane Protein Crystallization, L. DeLukas, Ed. (Elsevier Burlington, MA, 2009), vol. 63, pp 192-224.

32. Hunter, M.S. et al., X-ray diffraction from membrane protein nanocrystals. Biophys. $J$. in press.

33. Zaefferer, S. New developments of computer-aided crystallographic analysis in transmission electron microscopy. J. Appl. Cryst. 33, 10-25 (2000).

34. Murshudov, G. N., Vagin, A. A. \& Dodson, E. J. Refinement of macromolecular structures by the maximum-likehood method (1997) Acta Cryst. D53, 240-255. 
35. Praznikar, J., Afonine, P. V., Guncar, G., Adams, P. D. \& Turk, D. Averaged kick maps: less noise, more signal...and probably less bias", Acta Cryst. D 65, 921-931 (2009). 


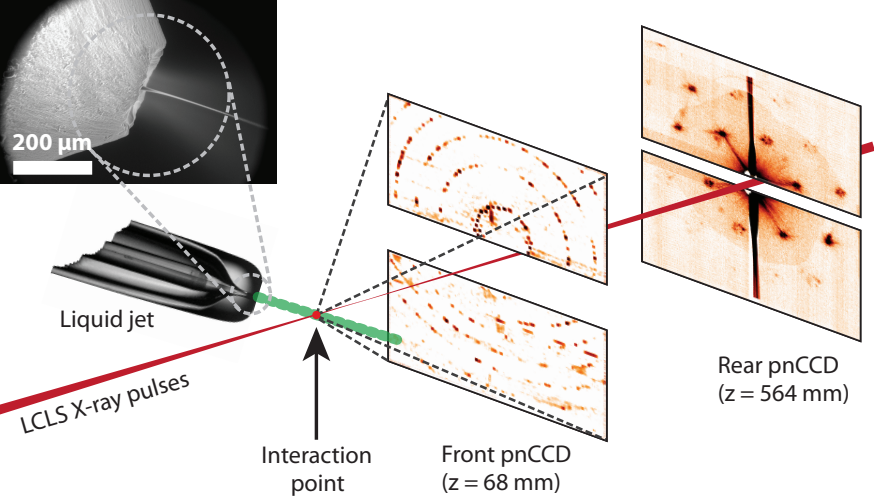


b

c $0 \overline{11}$

002
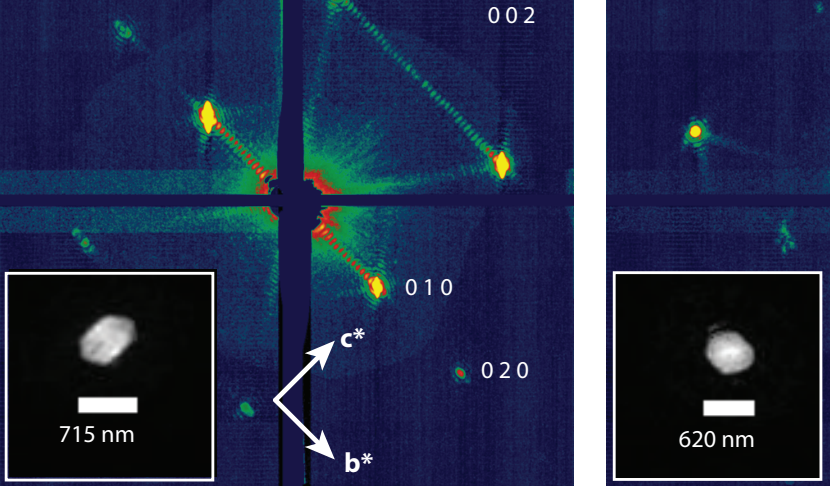

c)

C

d
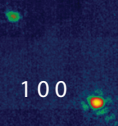

$0^{010}$

$\overline{1} 10$

\begin{tabular}{|c|}
\hline$a$ \\
$290 \mathrm{~nm}$
\end{tabular}

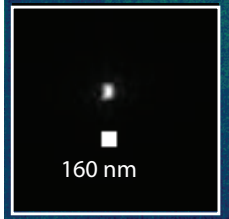



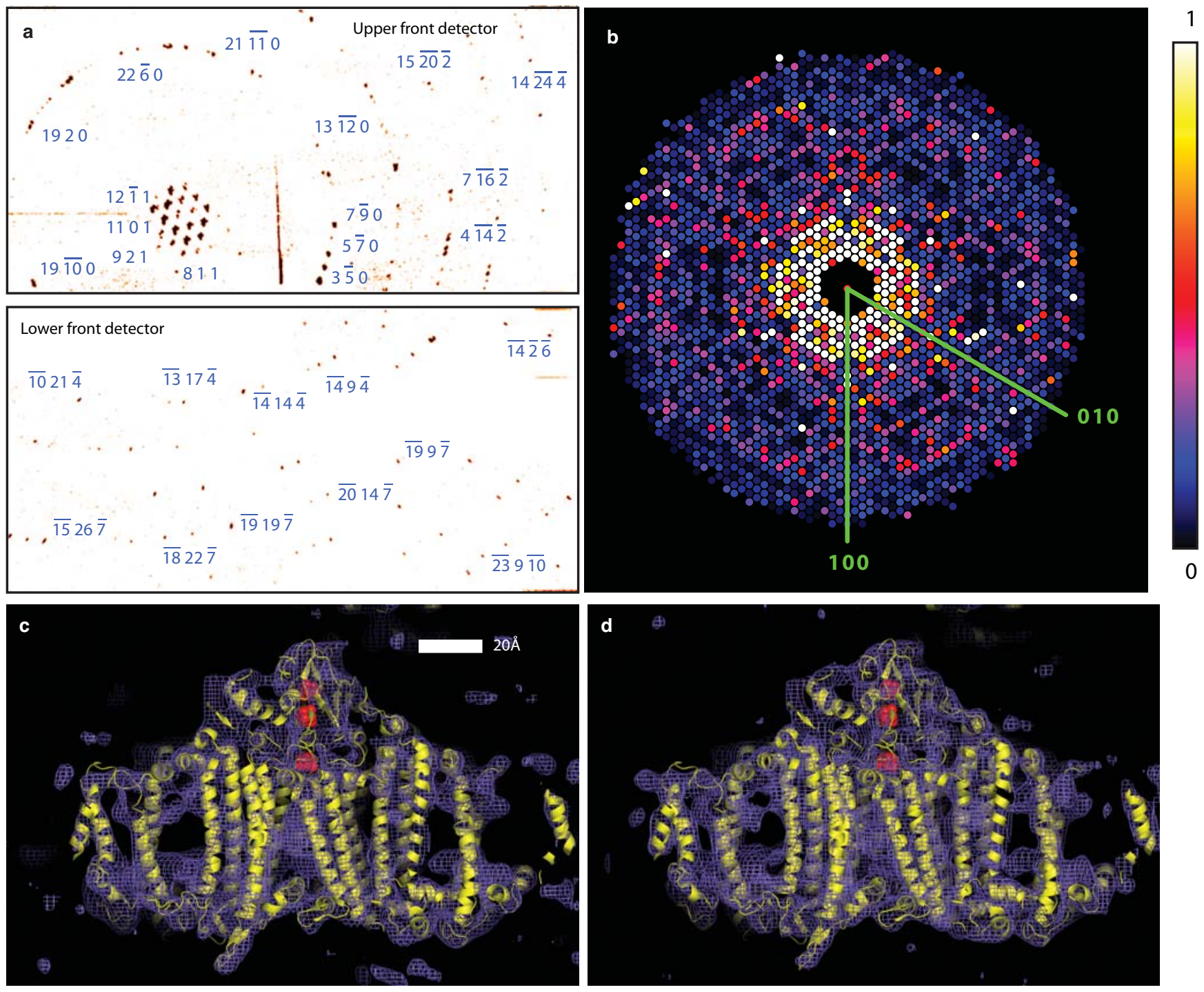


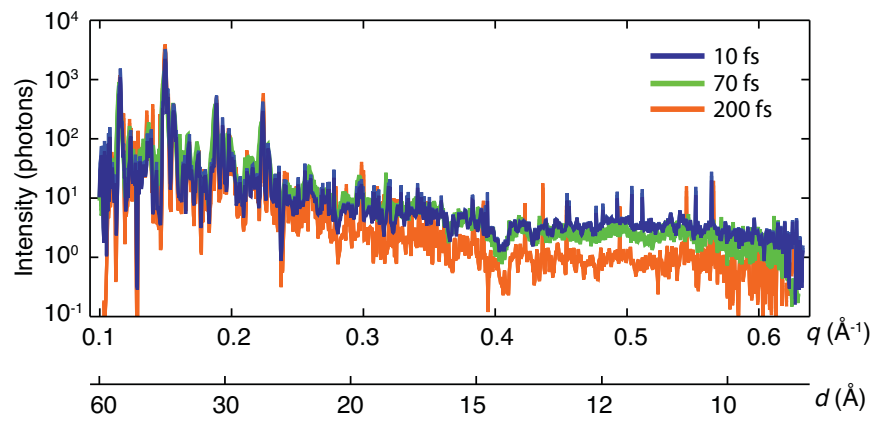

